\title{
Editorial for Special Issue "Applications of Synthetic Aperture Radar (SAR) for Land Cover Analysis"
}

\author{
John C. Trinder $D$ \\ School of Civil and Environmental Engineering, University of New South Wales (UNSW), \\ Sydney, NSW 2052, Australia; j.trinder@unsw.edu.au
}

Received: 24 July 2020; Accepted: 28 July 2020; Published: 29 July 2020

\begin{abstract}
Synthetic aperture radar (SAR) imaging systems derive microwave data, from space or airborne (piloted and remote piloted), that provide opportunities for the interpretation of many characteristics of the terrain surface. The increasing number of satellites equipped with SAR data acquisition systems that are being launched with a range of wavelengths, polarizations, and operating characteristics are enabling a better understanding of the earth's environment, for such activities as vegetation analysis, forest inventories, land subsidence, and urban analysis. In addition, airborne systems for remote piloted systems and ground-based systems are available. This Special Issue presents six quality scientific papers on typical applications of SAR technologies. They include methods for the determination of above ground biomass (AGB), crop mapping using data from an advanced X-band system developed in Japan, analysis of natural and human-induced slow-rate ground deformations in the region of Campania, in Italy, the location of landslides caused by natural phenomena based on SAR images derived from the Japanese high-resolution Advanced Land Observing Satellite-2 (ALOS-2), and monitoring the size of refugee camps and their environmental impacts caused by the displacement of people from Myanmar to the Cox's Bazar District, around Kutupalong, in Bangladesh. The paper concludes with some comments on the future directions of developments in SAR systems.
\end{abstract}

Keywords: SAR technologies; SAR remote sensing; SAR polarimetry; biomass measurement; vegetation monitoring; forest characteristics

\section{Introduction}

Radar technologies for mapping land cover and landform have been available since the 1960s, the systems originally being based on so-called real-aperture radar systems. Radar has the advantage for mapping tropical areas, because of its ability to penetrate cloud cover, and it can also operate during day or night. However, the resolution of the data derived from real aperture systems was poor and hence the mapping products were of low accuracy. The concept of the Synthetic Aperture Radar (SAR), which was known in the 1950s, is based on a moving platform, such as an aircraft or spacecraft. The distance the SAR antenna travels over a target, while the radar pulses are being returned to the antenna, creates a large synthetic aperture antenna, which can result in a much higher spatial resolution of the derived radar images, following the necessary processing of the raw data. The development of SAR for remote sensing applications commenced in 1960s, but research on the capabilities of SAR systems for remote sensing applications was not undertaken until 1978, with the launch of Seasat, and then missions planned for the Space Shuttle in the 1980s. Since then many missions have been developed by a range of countries with varying characteristics, including at least three different frequencies, namely, X-, C- and L-bands. Radar systems propagated electromagnetic waves which are often either dual or quad polarised. In addition, radar interferometry (InSAR), in which frequency differences in radiation emitted by one antenna and received by two separated antennas, can be used to measure height differences with high accuracy. This special issue, comprising six papers, describes 
some applications of the currently available SAR technologies for remote sensing and is a forerunner to applications that can be expected in the future as the technologies develop to include global coverage based on digital beamforming and multi-static systems, as well as radar tomography.

\section{Overview of Contributions}

In [1,2], methods were developed for determining above-ground biomass, the first being based on water cloud modelling (WCM) from quad-polarised L-band (PALSAR) data on the Advanced Land Observing Satellite-2 (ALOS-2), and the second from dual-polarised X-band TerraSAR-X and quad-pol-InSAR C-band data from RADARSAT-2 data. The concept of water cloud modelling (WCM) was developed in [3], in which a vegetation canopy was represented by a water cloud, based on a number of parameters which were obtained through regression analysis of the radar cross section data for ground truth data based on four crop types. A theoretical study was carried out in [4], in which it was found that the model will be helpful to interpret SAR signals in vegetated areas; however, since there are variabilities in the parameters of the model, it is still difficult to use the WCM without in situ experimental data.

The authors in [1] discuss the options for the decomposition of quad-pol SAR data and the retrieval of scattering components of forest vegetation. The shift in the polarization orientation angle (POA) affects the PolSAR-based scattering information, which is dependent on the Faraday rotation angle (FRA) and structural properties of the scatterer. An extension of the WCM used in this paper reduces the need for calibration from field data because it retrieves ground and vegetation scattering from polarimetric decomposition and the ambiguity in the scattering due to the POA shift is compensated in coherency matrix elements.

The paper uses the Yamaguchi four-component scattering model, which comprises surface, double-bounce, volume, and helix scattering, where volume and helix scattering are merged to represent the vegetation scattering component. Ground scattering is represented by surface scattering, while ground-stem scattering is represented by double bounce. The WCM developed by [3] assumes that the vegetation is composed of uniformly distributed water droplets, which is an over-simplification of reality. It has subsequently been modified by other researchers, while the authors in this paper have included terms that consider possible interaction of the SAR signal with various components of forests, such as scattering through the gaps between the forest vegetation, ground backscatter with the attenuation of the electromagnetic waves through the forest canopy, backscattering through the vegetation layer with a transmissivity of the electromagnetic waves of less than one, and the forest transmissivity of the electromagnetic waves in the presence of an attenuating layer of the forest. The above-ground biomass (AGB) of a resolution cell can be determined from the formulation of the total backscatter. Higher order scattering mechanisms are also represented in the paper.

The study area is a northern semi-evergreen tropical forest in Dudhwa National Park in northern India, in which the full statistics of the trees were determined in the field. Fully polarimetric ALOS-2 data were processed for the evaluation of the POA shift due to the Faraday rotation. The results of the tests on forest plots in the Dudhwa National Park using quad-pol data revealed that the data had been accurately compensated for the Faraday rotation errors by the Japan Aerospace Exploration Agency (JAXA). Regression of field based AGB and backscatter revealed the need to apply deorientation of the coherency matrix. Unknowns for the determination of backscatter were obtained from decomposition modelling, while the backscatter values were used as the input to determine AGB. Graphs were presented for the values of AGB from field and the modelling. An analysis of the regression of modelling of AGB against field determinations for the forest plots of mixed and single tree species revealed that, after POA compensation, $\mathrm{R}^{2}$ varied from 0.86 to $<0.1$ and AGB 74 to $18 \mathrm{t} / \mathrm{ha}$. Comparisons were made with several other published results. The paper claims that the use of the extended WCM (EWCM) requires a minimum dependence on field measurements to determined AGB. The paper concludes with the recommendation for "deorientation procedure before polarimetric decomposition to minimize the problem of overestimation of volume scattering and underestimation of double-bounce scattering". 
In [2], the authors determined canopy height and above-ground biomass in a peat swamp tropical forest, with an area of 4340 ha, in central Kalimantan, on the island of Borneo, Indonesia, using pol-InSAR data. Airborne lidar data, with a point spacing of 10.7 points $/ \mathrm{m}^{2}$ and drone photography with a ground sampling distance of $5 \mathrm{~cm}$, were derived over all field inventory areas, where DBH, tree height, and species of sample trees were determined. SAR data collected were C-band from Radarsat-2 (RS-2) quad-pol data in ascending and descending modes and X-band dual-pol data from TerraSAR-X (TS-X) satellite in ascending mode.

Canopy height was determined by subtracting the digital terrain model (DTM), derived from filtered lidar point cloud from the digital surface model from the highest points in the lidar point cloud. The AGB could be determined from the heights of the trees, DBH, and wood specific density, which were determined by the field inventory, with different allometric models being used according to tree DBH. In addition, the drone photography was automatically processed to derive a DSM. The canopy height model determined from the elevations from the drone photography was proved to correlate well with the canopy height model determined by the lidar.

The SAR data were filtered to reduce the effects of the speckle, repeat passes were co-registered, and filtered interferograms computed. Two inversion models were tested to estimate canopy height from the SAR data, 'Random Volume over Ground' (RVoG) interferometric model, and the 'Random Motion over Ground' (RMoG). The paper states:

- "RVoG model is a simple two-layer model, in which one layer represents the forest canopy and the other a reflective ground layer below the vegetation layer. It simulates vegetation as a homogeneous layer of fixed thickness containing volume scatterers with randomly oriented particles over a ground scatterer. The model ignores the even-bounce scattering mechanism as well as higher order interactions."

- "RMoG model separates temporal and volumetric decorrelations into four structural parameters and two dynamic parameters. The structural parameters are the tree height, wave extinction, ground topography and ground-to-volume ratio. The dynamic parameters are known as ground and canopy motion standard deviations induced by the temporal baseline."

The tests of the suitability of RVoG and RMoG for estimating canopy height were based on the drone DSM and the lidar DTM as reference. They revealed that RVoG strongly overestimated the canopy heights and therefore RMoG was superior to RVoG. The estimation of the canopy heights from RS-2 and TS-X by Pol-InSAR tended to overestimate the canopy height in lower heights and underestimate them in forested areas. However, a comparison of the results from the two SAR satellites indicated that elevations derived from TS- $X$ tended to strongly underestimate the heights compared to heights derived from RS-2 data. A saturation effect occurred at about $15 \mathrm{~m}$ for TS-X data, and at about $20 \mathrm{~m}$ for RS-2 data. Overall, the study revealed that data from both satellites are suitable for determining canopy height, but data from these satellites may not be suitable in some cases, due to issues with decorrelation. Correlations between AGB derived by lidar and both sets of satellite data revealed coefficients of determination of $0.81-0.85$ for RS- 2 and of $0.60-0.85$ for TS-X, but both sensors tended to overestimate the AGB in lower biomass ranges and underestimate AGB in higher biomass ranges. Biomass maps for the region derived from Sentinel-2, TS-X and two previous studies were presented for comparison.

The Advanced Satellite with New system ARchitecture for Observation-2 (ASNARO-2), with an X-band SAR (XSAR) on board [5], was launched in January 2018 by Nippon Electric Company (NEC), Japan, featuring very high resolution imagery in Spotlight mode (1.0 $\mathrm{m}$ spatial resolution). The data in Spotlight mode were evaluated for their potential use both on their own and in combination with Sentinel-1 C-band SAR data for generating crop maps. Selected radar indices were also considered in the paper. Machine learning algorithms considered were SVM with Gaussian kernel function, random forests, the extreme learning machine (ELM) with a Gaussian radial basis function (RBF) kernel, and a multilayer feedforward neural network (FNN). Bayesian optimization was used to determine the hyperparameters in these operations. 
The study area is in the town of Memuro, Hokkaido, northern Japan, where five vegetable crops (beans, beetroot, maize, potato, and winter wheat) with varying harvest times were studied for classification potential. ASNARO-2 has a sun-synchronous orbit at an altitude of $504 \mathrm{~km}$ with one day repeat cycle over Japan. Two scenes were acquired in Spotlight mode with HH polarization in descending mode. Two ascending Sentinel-1B C-band Interferometric Wide-Swath (IW) scenes with VH/VV polarization were also obtained. Data were orthorectified using the $10 \mathrm{~m}$ mesh DEM produced by the Geospatial Information Authority of Japan (GSI) for the Earth Gravitational Model 2008.

The Jeffries-Matusita (J-M) distances were used to determine the separability of crop types. Therefore, the following three cases were investigated: Case 1, X-band HH polarization data from ASNARO-2 XSAR combined with C-band VH/VV polarization data from Sentinel-1B; Case 2, X-band $\mathrm{HH}$ polarization data from ASNARO-2 XSAR; Case 3, C-band VH/VV polarization data from Sentinel-1B. For the classifications described above, the data were divided into three parts: $50 \%$ for training, $25 \%$ for evaluation, and $25 \%$ to assess the generalization errors in the final selected model. The procedures were repeated 10 times. A full range of parameters, describing the classification accuracies, were determined and statistical testing was carried out to determine whether the results of the different classification methods were significantly different. The results indicated that separability of crops based on the J-M distances were much clearer for the ASNARO-2 XSAR data than on the Sentinel-1B data.

Regarding the classification accuracies, all four methods gave improved results when the X-SAR and C-band SAR were used in combination. SVM achieved the highest overall accuracy of $0.85 \pm 0.018$ $(\mathrm{kappa}=0.810 \pm 0.023 ; \mathrm{AD}+\mathrm{QD}=0.146 \pm 0.018)$ "quantity disagreement $(\mathrm{QD})$, allocation disagreement (AD)". While all methods achieved overall accuracies above 0.8 , the differences were shown to be statistically significant. Overall, the accuracies achieved indicated that combining data from ASNARO-2 XSAR with data from larger satellites was advantageous in terms of achievable accuracies. The combination of the two data types led to fewer misclassifications. Full details of accuracies achievable with the tests are available in the paper.

A comprehensive analysis of natural and human-induced slow-rate ground deformations over the period from 1992 to 2010 in the region of Campania, Italy was undertaken in [6] by the Persistent Scatterer Interferometry (PSI) approach, using archived datasets. The Campania region is a tectonically active area, as well as being affected by anthropogenic causes of surface deformations. These deformations vary from an uplift of about $0.25 \mathrm{~mm} / \mathrm{yr}$ in the southwestern coastal sector, to south westward velocities between 2 and $4 \mathrm{~mm} / \mathrm{yr}$ at Vesuvius and the island of Ischia at the southwestern corner of the Campanian Plain, which were based on measurements by levelling lines and GNSS (Global Navigation Satellite Systems) data. The paper provides a brief history of earlier efforts to determine surface deformations by interferometric methods, as well as the geology of the region.

The paper described the PSI method as being based on the use of a long series of co-registered, multi-temporal SAR image data for the analysis of coherent pixels over a sequence of interferograms and for the production of maps of displacement velocities measured along the line of sight (LOS). These maps show the temporal changes in ground displacement for each SAR epoch, which can be presented as annual average displacement velocity over the entire acquisition period, or as relative displacements with respect to a reference at zero time in a series.

For this paper, six PSI data sets were obtained comprising ascending and descending orbits from ENVISAT, RADARSAT, and ERS-1/2, acquired from June 1992 to December 2001 for ERS-1/2, from November 2002 to July 2010 for ENVISAT, partly overlapping with RADARSAT (March 2003 to September 2007). The PSI datasets comprise about 5.8 million PS, which were identified within the study area with a coherence higher than 0.65 . They are characterized by negative mean displacement velocities in both the ascending and descending orbit datasets. The ERS and ENVISAT datasets were processed with PSP (Persistent Scatterer Pair Interferometry), while the PS-InSAR method was only available for RADARSAT data. The PSP method overcomes problems due to the presence of slowly varying artefacts in the phase, due to the atmospheric and orbital effects, by relying only on the relative 
signal in pairs of points close enough to be affected by the same disturbance. Therefore, the PSP technique is robust for spatially correlated disturbances and does not require preliminary processing.

Each point of the PSI datasets is identified by (a) identifier code; (b) North and East coordinates; (c) time series of measurements (expressed in $\mathrm{mm}$ ) of the displacement along the LOS; (d) average velocity expressed in $\mathrm{mm} / \mathrm{yr}$; (e) standard deviation of the average velocity; (f) coherence, which is a normalized index of the local signal-to-noise ratio of the interferometric phase and reflects the accuracy of PS measurements. Accurate velocities of $1 \mathrm{~mm} / \mathrm{yr}$ along the LOS are possible with PSI when many SAR images are used, assuming a coherence threshold value of the PS greater than 0.65 . The LOS displacement rates vary from -24.5 to $+15.2 \mathrm{~mm} / \mathrm{yr}$ for ERS datasets, -51.1 to $+30.7 \mathrm{~mm} / \mathrm{yr}$ for RADARSAT datasets, and -10.6 to $+6.0 \mathrm{~mm} / \mathrm{yr}$ for ENVISAT datasets.

Average velocities were calculated by averaging the LOS velocities derived from ascending and descending LOS velocities and they were then converted to East and height values, (Northern displacements are not available due to the N-S direction of the orbits of the satellites) using the look angles for the data acquisitions. The results are presented in $100 \mathrm{~m}$ spaced grid maps showing the accumulated distribution of vertical and E-W movements in the Campania territory (expressed in $\mathrm{mm} /$ year) for the whole period of 1992-2010.

The derived ground deformation vertical and E-W horizontal components for an area of $12,160 \mathrm{~km}^{2}$, represent $89.5 \%$ of the whole Campania territory, subject to a variety of environmental, topographic and geological conditions. Assessments were made of the characteristics of the displacements across the region in considerable detail. The dominant sources of deformations vary with location, which are demonstrated by the maps. In conclusion the paper demonstrates the importance of using PSI data within tectonically active areas. Future missions, especially with X-band SAR, should be used for regular assessments of the Campania territory, to develop a more complete and detailed understanding of anthropogenic and natural deformations.

In [7] SAR images derived from the Japanese high-resolution Advanced Land Observing Satellite-2 (ALOS-2) were investigated to rapidly locate landslides caused by natural phenomena, such as earthquakes or heavy rainfall. SAR data have the advantage that they can penetrate clouds and are independent on illumination by sunlight. The study site is at Iburi, Hokkaido, Japan, which suffered a 6.6 Mw earthquake at a depth of $35 \mathrm{~km}$ in 2018. The earthquake triggered a total of 6000 landslides over an area of $400 \mathrm{~km}^{2}$ where the affected terrain had elevations from 100 to $250 \mathrm{~m}$, and slope gradients of $15-35^{\circ}$. Three overlapping SLC ALOS-2 L-band HH polarised images, two pre-events, and one post-event were available for the research, comprising phase and amplitude with a sampling spacing in range and azimuth of $1.43 \mathrm{~m} \times 1.95 \mathrm{~m}$, respectively. A detailed landslide inventory map was created from a combination of manual extraction and details held by Geospatial Information Authority of Japan (GSI).

Parameters derived from pre- and post-event SAR ALOS-2 images for identification of landslides included intensity difference and post-event correlation coefficient, as well as the differences between pre- and post-event correlations, for cases when the correlation coefficient is consistently low. Coherence based on the cross-correlation of phase information in two images was also calculated, as well as the differences between pre-event and post-event coherence. The parameters were investigated using window sizes ranging from $5 \times 5$ to $85 \times 85$ pixels for pixel sizes of approximately $3 \mathrm{~m}$. Landslides ranged from hundreds to tens of thousands metres ${ }^{2}$ in area.

Qualitative assessments were carried out to assess the effectiveness of the computed parameters by overlapping the landslide ground truth data on images displaying the calculated parameters. Intensity differences clearly showed lower and higher value pixels in landslide areas, since landslides smoothed hillsides, causing backscattering decrease, and roughened foothill areas that induced backscattering increases. Post-event correlation coefficient and correlation coefficient differences displayed lower value pixels in landslide regions due to larger ground changes. Post-event coherence and coherence difference also showed some lower value pixels in landslide areas owing to the decorrelation in the ground changes caused by landslides. 
The quantitative assessments of the parameters were achieved by deriving receiver operating characteristic (ROC) curves, designed for illustrating the diagnostic ability of binary classifiers, 0 as in [8], by displaying true positive rate (TPR) values against false positive rate (FPR) values. Intensity related parameters were found to be more stable in the presence of minor ground changes due to landslides and thus showed better performance than coherence related parameters. Intensity difference and correlation coefficient differences performed better as landslide and non-landslide pixel classifiers and are recommended for future applications. Individually, other parameters are not very appropriate as binary classifiers for distinguishing landslide and non-landslide pixels. The paper identified several parameters for their suitability for identifying landslide and non-landslide parameters, but the best accuracies of the classifications were relatively low at $64-69 \%$ for landslide and non-landslide pixels, derived by simple ROC thresholds. The combined application of the intensity difference, correlation coefficient, and coherence parameters through linear discriminant analysis achieved an overall accuracy of around $74 \%$. It should be observed however, that this mapping task was based on only three scenes, two pre-events, and one post-event, in a region comprising massive shallow landslides on small to medium slopes covered by trees. More work would be required to determine which parameters provide conclusive evidence on the classification of landslides.

In [9], the changes in the area of refugee camps and consequent environmental effects caused by the displacement of people from Myanmar to the Cox's Bazar District, around Kutupalong, in Bangladesh, close to the border of Myanmar, were monitored, including massive decreases of forest cover and agricultural lands, which were converted to areas for housing, sanitation, and infrastructure facilities. The paper commenced with details of reports and surveys on the historical development of the camp and its significant effects on natural resources, ecosystems, and the health of the refugees. The limitations of the previous surveys included the use of pixel-based image analysis methods, such as NDVI, which led to scale errors in the analysis of multiple images. Advantages of SAR images for the analysis of the impacts of the camp include penetration of cloud cover, as well as acquisition costs and the increased information content. A new method of assessing the anthropogenic impacts on the environment was also developed for this paper, which had the following aims, to: (1) develop a framework based on radar images; (2) maintain objectivity at the selection of reference areas; (3) perform a multi-scale approach to reduce the scale-dependency of the generated change detection; (4) make use of polarimetric information using two SAR sensors of different wavelength and polarizations.

The paper furthermore reveals which wavelengths, polarimetric features, and spatial resolutions are most suitable to describe landscape changes at the local to regional scale.

Sentinel-1 (S1) C-band dual-polarized, and quad-polarized ALOS-2 (A2) L-band images were used for monitoring the Cox's Bazar area. C-band S1 with a wavelength of $5.5 \mathrm{~cm}$ is strongly sensitive to the roughness of canopies and moisture of non-vegetated surfaces, while L-band A2 with a wavelength of $22.9 \mathrm{~cm}$, is able to partly penetrate surfaces and reveal structures under canopies, such as stems or branches of trees. To manage variations in phenology in the vegetation, SAR image pairs of S1 and A2 with the same flight direction for eleven dates between 2016 and March 2019 were selected. Training areas were selected from Landsat 7 and 8 and Sentinel 2 optical images according to acquisition date, cloud cover, and coverage of the study area, using a range of indices. A classification and regression tree (CART) was processed to retrieve a coarse pre-classification of four classes (vegetation, water, open, and camp) in the optical images used for training for which accuracies were between $87.3 \%$ and $96.9 \%$.

The characteristics of the topography were determined from SRTM $30 \mathrm{~m}$ data comprising elevations, slope, north and east aspects, plan curvature, and profile curvature and a classification of morphometric features. The data were resampled to a spatial resolution of $10 \mathrm{~m}$ to match the resolution of the final classification. Since these parameters are highly scale-dependent, this analysis was performed for window sizes ranging from $1 \times 1$ to $49 \times 49$ pixels.

The radar images were calibrated using relevant meta data, terrain flattened Gamma0 was derived to minimize the impact of the incidence angle on backscatter intensity, and the effects of speckle were reduced. All images were co-registered to a stack based on their orbit information and geocoded 
using range Doppler terrain correction. The resulting data therefore for each date consisted of four polarizations (HH and HV from ALOS-2; VH and VV from Sentinel-1) at a common spatial resolution of $10 \mathrm{~m}$. The radar images were filtered using a median filter with window sizes ranging from $1 \times 1$ to $49 \times 49$ pixels and then processed for the extraction of 8 indices leading to 126 features per investigated date (84 radar features and 42 constant terrain features). A random forest classifier was selected to identify the features that are most suitable for the description of the study area using 150 decision trees and one third of the available training pixels per class.

The results indicated that the highest increase in the area of the camp was observed between July 2017 and February 2018, when it increased tenfold. There were also significant seasonal effects on the areas of vegetation including due to phenology, as well as in the open and in water areas. It was possible to trace the growth in the camp size as well as the loss of vegetation over the study period. Assessment of the accuracy of derived maps was based on independently collected validation points, which were sampled according to specific rules and amounted to 576 points for each date. Overall accuracies varied from $88.2 \%$ to $95.1 \%$ at an average of $92.1 \%$. The paper states: that 'the classified vegetation cover can be trusted throughout the study to a high degree, which was additionally confirmed by the kappa values between 0.8 and 0.9.' Camp areas were classified with very high kappa values and low errors of commission and omission. This means the expansion of the camps and the accompanying decrease of vegetation not only corresponded to the values found in other studies, but they were also well described by SAR data. The ambiguity in the backscatter intensity of solid and light dwellings can become a problem in studies dealing with humanitarian settings, but more research is required on this topic. The importance of various features was then discussed in the paper.

In conclusion, the use of SAR images increased the quality of the land cover assessments, as they allowed for input images in the wet seasons, which helped us to consider the variations in phenology. This increased the quality of land cover classifications, because SAR images enabled the assessment of physical surface characteristics, instead of spectral reflectance. Vegetation was found to be characterized best by the Volume Scattering Index (VSI), the Radar Forest Degradation Index (RFDI), and a combined index. Topography played a role in the identification of classes, especially in cases where large areas were covered by homogenous classes, such as water bodies and large evergreen forest areas. All classified dates showed high classification accuracies, especially for water and camp areas.

In a humanitarian context, the integration of SAR data not only gives higher flexibility, in respect of the number of images and the definition of suitable acquisition dates, but also allows for the identification of landcover types by characteristics of surfaces (size, form, roughness, material, and scattering mechanisms). Therefore, SAR images are a valuable source of information for future emergencies, now that sufficient studies exist to demonstrate their applicability.

\section{Conclusions}

While the six papers demonstrate some interesting applications of SAR image data, there are many more applications of SAR image data that could be presented. Examples of the application of new sources of data, such as P-band and S-band, would be of interest to readers. Furthermore, deep learning approaches to image interpretation for vegetation and urban analysis were not provided in this special series, nor were the exploration of data that could be derived by advanced SAR technologies, such as multi-static radar systems or radar tomography. Radar technologies are advancing rapidly, and future developments will have a significant impact on the type of data that will become available in the next decade for remote sensing applications.

Funding: This research received no external funding.

Conflicts of Interest: The author declare no conflict of interest. 


\section{References}

1. Kumar, S.; Garg, R.D.; Govil, H.; Kushwaha, S.P.S. PolSAR-Decomposition-Based ExtendedWater Cloud Modeling for Forest Aboveground Biomass Estimation. Remote Sens. 2019, 11, 2287. [CrossRef]

2. Berninger, A.; Lohberger, S.; Zhang, D.; Siegert, F. Canopy Height and Above-Ground Biomass Retrieval in Tropical Forests Using Multi-Pass X- and C-Band Pol-InSAR Data. Remote Sens. 2019, 11, 2105. [CrossRef]

3. Attema, E.P.W.; Ulaby, F.T. Vegetation modeled as a water cloud. Radio Sci. 1978, 13, 357-364. [CrossRef]

4. Park, S.-E.; Jung, Y.T.; Cho, J.-H.; Moon, H.; Han, S. Theoretical Evaluation of Water Cloud Model Vegetation Parameters. Remote Sens. 2019, 11, 894. [CrossRef]

5. Sonobe, R. Combining ASNARO-2 XSAR HH and Sentinel-1 C-SAR VH/VV Polarization Data for Improved Crop Mapping. Remote Sens. 2019, 11, 920. [CrossRef]

6. Matano, F. Analysis and Classification of Natural and Human-Induced Ground Deformations at Regional Scale (Campania, Italy) Detected by Satellite Synthetic-Aperture Radar Interferometry Archive Datasets. Remote Sens. 2019, 11, 822. [CrossRef]

7. Ge, P.; Gokon, H.; Meguro, K.; Koshimura, S. Study on the Intensity and Coherence Information of High-Resolution ALOS-2 SAR Images for Rapid Massive Landslide Mapping at a Pixel Level. Remote Sens. 2019, 11, 808. [CrossRef]

8. Fawcett, T. An introduction to ROC analysis. Pattern Recognit. Lett. 2006, 27, 861-874. [CrossRef]

9. Braun, A.; Fakhri, F.; Hochschild, V. Refugee Camp Monitoring and Environmental Change Assessment of Kutupalong, Bangladesh, Based on Radar Imagery of Sentinel-1 and ALOS-2. Remote Sens. 2019, 11, 2047. [CrossRef]

(C) 2020 by the author. Licensee MDPI, Basel, Switzerland. This article is an open access article distributed under the terms and conditions of the Creative Commons Attribution (CC BY) license (http://creativecommons.org/licenses/by/4.0/). 